\title{
Proteasome inhibition protects against diet-induced gallstone formation through modulation of cholesterol and bile acid homeostasis
}

\author{
EUN-JI LEE ${ }^{1}$, MIN HEE KIM ${ }^{1}$, YE-RYUNG KIM ${ }^{2}$, JOO-WON PARK ${ }^{2}$ and WOO-JAE PARK ${ }^{1}$ \\ ${ }^{1}$ Department of Biochemistry, School of Medicine, Gachon University, Incheon 21999; ${ }^{2}$ Department of Biochemistry, \\ College of Medicine, Ewha Womans University, Seoul 07985, Republic of Korea
}

Received February 9, 2017; Accepted December 7, 2017

DOI: $10.3892 /$ ijmm.2017.3326

\begin{abstract}
Gallstone disease is one of the most prevalent and costly gastrointestinal disorders worldwide. Gallstones are formed in the biliary system by cholesterol secretions in bile, which result from excess cholesterol, a deficiency in bile salts or a combination of the two. The present study examined the effects of proteasome inhibition on gallstone formation using the proteasome inhibitors bortezomib (BT) and carfilzomib (CF). C57BL/6J mice were fed a lithogenic diet to generate gallstones and injected with BT or CF for 12 weeks. After 12 weeks of the lithogenic diet, 8 out of the 10 mice in the control group had developed gallstones, whereas none of the mice who received proteasome inhibitors had developed gallstones. Notably, the expression of genes associated with cholesterol synthesis (sterol regulatory element-binding protein-2 and 3-hydroxy-3-methylglutaryl-CoA reductase), cholesterol secretion [ATP-binding cassette subfamily $\mathrm{G}$ member $5(A B C G 5)$ and $A B C G 8]$ and bile acid synthesis
\end{abstract}

Correspondence to: Dr Woo-Jae Park, Department of Biochemistry, School of Medicine, Gachon University, Incheon 21999, Republic of Korea

E-mail: ooze@gachon.ac.kr

Dr Joo-Won Park, Department of Biochemistry, College of Medicine, Ewha Womans University, Seoul 07985, Republic of Korea

E-mail: joowon.park@ewha.ac.kr

Abbreviations: ABCG, ATP-binding cassette transporter; BSEP, bile salt export pump; Cyp7a1, cholesterol $7 \alpha$-hydroxylase; Cyp7b1, oxysterol 7 $\alpha$-hydroxylase; Cyp27a1, sterol 27-hydroxylase; Cyp8b1, sterol 12 $\alpha$-hydroxylase; ERK, extracellular signal-regulated kinase; FXR, farnesoid X receptor; GAPDH, glyceraldehyde 3-phosphate dehydrogenase; HMGCR, 3-hydroxy-3-methylglutaryl-CoA reductase; JNK, c-Jun $\mathrm{N}$-terminal kinase; LXR, liver $\mathrm{X}$ receptor; MDR, multidrug resistance; PKA, protein kinase $\mathrm{A}$; PXR, pregnane $\mathrm{X}$ receptor; SHP, small heterodimer partner; SREBP, sterol regulatory element-binding protein

Key words: gallstone disease, proteasome inhibition, cholesterol synthesis, bile acid synthesis, lithogenic diet, cholesterol secretion [cytochrome P450 family 7 subfamily A member 1 (Cyp7al), Cyp7b1, Cyp27al and Cyp8b1] was reduced in the livers of mice injected with BT or CF. Cyp7al encodes cholesterol $7 \alpha$-hydroxylase, the rate-limiting enzyme in the synthesis of bile acid from cholesterol. The present study therefore measured the expression levels of transcription factors that are known to inhibit Cyp7al expression, namely farnesoid $X$ receptor $(F X R)$, pregnane $X$ receptor (PXR) and small heterodimer partner (SHP). Although FXR, PXR and SHP expression was predicted to increase in the presence of proteasome inhibitors, the expression levels were actually reduced; thus, it was concluded that they were not involved in the proteasome inhibition-induced regulation of Cyp7al. Further investigation of the mitogen-activated protein kinase and protein kinase A (PKA) signaling pathways in human hepatoma cells revealed that proteasome inhibition-induced c-Jun N-terminal kinase (JNK) phosphorylation reduced CYP7A1 and CYP27Al expression. In addition, reduced PKA phosphorylation as a result of proteasome inhibition regulated ABCG5 and ABCG8 expression. In conclusion, these findings suggest that proteasome inhibition regulates cholesterol and biliary metabolism via the JNK and PKA pathways, and is a promising therapeutic strategy to prevent gallstone disease.

\section{Introduction}

Gallstone disease, the most common biliary disorder affecting $10-15 \%$ of the adult population, is a significant health problem worldwide, with an incidence that has been increasing due to the rising epidemic of obesity and metabolic syndrome (1). Bile contains cholesterol, bile salts and phospholipids, and the precipitation of excessive cholesterol in bile leads to gallstone formation (2). To overcome the extremely low solubility of cholesterol in aqueous solutions, cholesterol in bile exists as mixed micelles of bile salts and phospholipids. Excess cholesterol, a lack of bile salts or phospholipids, or a combination of these factors can lead to the supersaturation of bile and the precipitation of cholesterol $(2,3)$. Therefore, cholesterol homeostasis is required to prevent gallstone formation.

The balance between cholesterol intake/biosynthesis and biliary cholesterol secretion is important for cholesterol homeostasis (4). The master regulator of cholesterol biosynthesis, 
sterol regulatory element-binding protein-2 (SREBP-2), regulates the expression of numerous genes involved in cholesterol homeostasis, including the rate-limiting enzyme in cholesterol biosynthesis, 3-hydroxy-3-methylglutaryl-CoA reductase (HMGCR) (4). In addition, the cytochrome P450 genes, cytochrome P450 family 7 subfamily A member 1 (CYP7A1), CYP7B1, CYP27A1 and CYP8B1, encode enzymes that catalyze various steps in cholesterol catabolism and bile acid synthesis. Transcriptional regulation of CYP7A1, which encodes cholesterol $7 \alpha$-hydroxylase and catalyzes the initial rate-limiting step in cholesterol catabolism (5), has been relatively well-established. The farnesoid X receptor (FXR) induces expression of an atypical orphan nuclear receptor, small heterodimer partner (SHP), which then inhibits liver-related homolog-1 transactivation, thereby inhibiting Cyp7al transcription (5). In addition, SHP and the pregnane $X$ receptor (PXR) interact with hepatocyte nuclear factor $4 \alpha$ and block its recruitment of peroxisome proliferator-activated receptor- $\gamma$ coactivator- $1 \alpha$ to $C Y P 7 A 1$ chromatin, thereby inhibiting CYP7Al transcription (5). Finally, the ATP-binding cassette $(\mathrm{ABC})$ transporters $\mathrm{ABCB} 4$ [also known as multidrug resistance protein $2(\mathrm{Mdr} 2)$ ], ABCB11 [also known as bile salt export pump (Bsep)] and ABCG5/ABCG8 heterodimers serve essential roles in the biliary secretion of phospholipids, bile acids and cholesterol, respectively (6). Regulation of cholesterol metabolism is a therapeutic target, and statins are reported to be effective to treat gallstone disease (7) and to reduce the risk of cholecystectomy (8). However, the efficacy of statin treatment for gallstone diseases remains under debate (9). Ursodeoxycholic acid (UDCA) was Food and Drug Administration (FDA) approved for cholesterol gallstone dissolution through the increase in bile flow and the alteration of the hydrophobicity index of the bile acid pool (10). However, UDCA use in neonatal and infant cholestasis was reported to be ineffective and unsafe, and it can also cause severe toxicities, including hepatitis, pruritus, cholangitis, ascites, vanishing bile duct syndrome and liver failure, in adults (10). Therefore, novel drugs to treat gallstone disease are required to apply to the range of patients.

The proteasome, which degrades ubiquitinated proteins, has emerged as an attractive therapeutic target to treat various cancer types (11). Bortezomib (BT), the first FDA-approved proteasome inhibitor, has been used clinically to treat patients with multiple myelomas and mantle cell lymphoma (12). Moreover, BT has been under investigation to expand its therapeutic effects to various other conditions, including fatty liver (13), hepatic injury by ductal ligation (14), drug-induced liver injury (15), pancreatic cancer (16), breast cancer (17), renal cell carcinoma (18) and prostate cancer (19). In addition, other proteasome inhibitors, including carfilzomib (CF), CEP-18770, PR-047 and NPI-0052, have also been developed and tested for the treatment of a variety of diseases (20).

The present study investigated the effects and mechanism of proteasome inhibition in gallstone formation using two proteasome inhibitors, BT and CF by reducing cholesterol synthesis and biliary secretion. Furthermore, it was found that reduced transcription of Cyp7al by proteasome inhibition was associated with c-Jun N-terminal protein kinase (JNK) activation and that reduced ABCG5/ABCG8 levels resulted from reduced phosphorylation of protein kinase A (PKA).

\section{Materials and methods}

Materials. The following materials were purchased: i) SP600125, 8-bromo-cAMP, Sp-cAMPS and anti-Cyp7a1 (catalog no. SAB4301212) antibody (Sigma-Aldrich; Merck KGaA, Darmstadt, Germany); ii) PD98059 and anti-p38 (catalog no. 8690), anti-phosphorylated (phospho)-p38 (Thr180/Tyr182; catalog no. 4511), anti-JNK (catalog no. 9252), anti-p-JNK (Thr183/Tyr185; catalog no. 9255), anti-extracellular signal-regulated kinase (ERK; catalog no. 4695), anti-p-ERK (Thr202/Tyr204; 4370), anti-p-PKA (Thr197; catalog no. 5661) and anti-PKA (catalog no. 5842) antibodies (all Cell Signaling Technology, Inc., Danvers, MA, USA); iii) anti-SREBP-2 (catalog no. NV100-74543) antibody (Novus Biologicals, Littleton, CO, USA); iv) anti-Cyp27a1 antibody (catalog no. GTX103718; GeneTex, San Antonio, TX, USA); v) anti-HMGCR antibody (catalog no. ab174830) (Abcam, Cambridge, MA, USA); vi) anti-BSEP (catalog no. sc-74500), anti-MDR2 (sc-58221), anti-ABCG5 (catalog no. sc-517207) and anti-ABCG8 (catalog no. sc-30111) antibodies (all Santa Cruz Biotechnology, Inc., Dallas, TX, USA); vii) anti-glyceraldehyde 3-phosphate dehydrogenase (GAPDH) antibody (catalog no. MAB374) (EMD Millipore, Billerica, MA, USA); viii) anti-mouse-horseradish peroxidase (HRP; catalog no. 115-036-003) and anti-rabbit-HRP (catalog no. 111-035-003) antibodies (both Jackson Laboratory, Ben Harbor, ME, USA); and ix) BT and CF (both Biovision, Inc., Milpitas, CA, USA).

Animals and lithogenic diet provision. Male C57BL/6J mice $(\mathrm{n}=30 ; 6$ weeks old) were purchased from Orient Bio, Inc. (Seoul, South Korea) and were housed under special pathogen-free conditions. Experimental procedures were approved by the Animal Ethics Committee at Lee Gil Ya Cancer and Diabetes Institute of Gachon University, and all animals were treated in accordance with the Animal Care Guidelines of Lee Gil Ya Cancer and Diabetes Institute of Gachon University. Mice were housed in a controlled environment at $21-23^{\circ} \mathrm{C}$ and $51-54 \%$ humidity, with a $12 \mathrm{~h}$ light-dark cycle, and supplied with food and water ad libitum. Mice were fed a lithogenic diet (D12336; Research Diets Inc., New Brunswick, NJ, USA) and were injected with BT $(0.5 \mathrm{mg} / \mathrm{kg} /$ week $)$ or with CF $(0.5 \mathrm{mg} / \mathrm{kg} /$ week $)$ for 12 weeks. Control mice were injected with an equal amount of dimethyl sulfoxide. Mice were euthanized with low flow (10-30\% of total volume) of $\mathrm{CO}_{2}$, and sera, livers and gall bladders were collected and stored at $-80^{\circ} \mathrm{C}$ until further analysis.

Cell culture and treatment. Hep3B cells were purchased from the American Type Culture Collection (Rockville, MD, USA) and grown in Dulbecco's modified Eagle's medium supplemented with $10 \%$ fetal bovine serum and $1 \%$ penicillin/streptomycin (all Hyclone; GE Healthcare Life Sciences, Logan, UT, USA). The Hep3B cells were treated with $50 \mathrm{nM}$ BT, CF or epoxomicin (Epoxo) for $48 \mathrm{~h}$ along with $10 \mu \mathrm{M}$ SP600125 (JNK inhibitor), $10 \mu \mathrm{M}$ PD98059 (ERK inhibitor), $10 \mu \mathrm{M} 8$-bromo-cAMP (PKA activator) and $10 \mu \mathrm{M}$ Sp-cAMPS (PKA activator).

Western blotting. Liver and Hep3B cells were lysed with RIPA buffer [50 mM Tris-Cl (pH 7.5), $150 \mathrm{mM} \mathrm{NaCl}, 1 \%$ Nonidet 
P-40, $0.5 \%$ sodium deoxycholate and $0.1 \%$ SDS] containing protease and phosphatase inhibitors (Sigma-Aldrich; Merck $\mathrm{KGaA})$ at $4^{\circ} \mathrm{C}$ for $1 \mathrm{~h}$. Lysates were centrifuged at 12,000 x g, at $4^{\circ} \mathrm{C}$ for $10 \mathrm{~min}$, supernatants were collected and the protein concentrations of the supernatants were measured using Protein Assay Dye reagent (Bio-Rad Laboratories, Inc., Hercules, CA, USA). Proteins (50 $\mu \mathrm{g})$ were loaded and separated on $8-13 \%$ SDS-polyacrylamide gels, and then transferred to nitrocellulose membranes (Bio-Rad Laboratories, Inc.). Membranes were blocked with $5 \%$ bovine serum albumin (Sigma-Aldrich; Merck KGaA) in phosphate-buffered saline with $0.1 \%$ Tween-20 for $1 \mathrm{~h}$ at $4^{\circ} \mathrm{C}$ and then were incubated with primary antibodies (diluted 1:1,000) overnight at $4^{\circ} \mathrm{C}$. Membranes were then incubated with secondary antibodies (diluted 1:10,000) for $1 \mathrm{~h}$ at room temperature. Protein bands were detected using Enhanced Chemiluminescence Western Blotting Detection reagents (Amersham Biosciences, Little Chalfont, UK) and a ChemiDoc MP imaging system (Bio-Rad Laboratories, Inc.).

Cholesterol, phospholipid and bile acid measurements. Cholesterol, phospholipid and bile acid levels in sera, bile and livers were measured using the total cholesterol and cholesteryl ester colorimetric/fluorometric assay kit (Biovision, Inc.), the phospholipid assay kit (Sigma-Aldrich; Merck KGaA) and the total bile acids assay kit (Biovision, Inc.), respectively, according to the manufacturer's instructions.

Reverse transcription-quantitative polymerase chain reaction ( $q P C R)$. Total mRNAs from mouse livers were extracted with the NucleoSpin RNA II kit (Macherey-Nagel, Duren, Germany), and cDNAs were synthesized using a Verso cDNA Synthesis kit (Thermo Fisher Scientific, Inc., Waltham, MA, USA). qPCR was performed with SYBR-Green (Thunderbird; Toyobo Co., Ltd., Osaka, Japan) using a CFX Connect ${ }^{\mathrm{TM}}$ Real-Time PCR Detection system (Bio-Rad Laboratories). The thermal cycling conditions were as follows: $95^{\circ} \mathrm{C}$ for $1 \mathrm{~min}$, followed by 40 cycles of $95^{\circ} \mathrm{C}$ for $15 \mathrm{sec}, 60^{\circ} \mathrm{C}$ for $45 \mathrm{sec}$. Relative gene expression was calculated using the $2^{-\Delta \Delta \mathrm{Cq}}$ method (21). The primers that were used are described in Table I. GAPDH was used as the reference gene.

Statistical analysis. All of the experiments were repeated at least three independent times, and data are presented as the mean \pm standard error of the mean. Statistical significance was calculated using analysis of variance followed by Tukey's post hoc test (GraphPad Prism 6.0; GraphPad Software, San Diego, CA, USA). $\mathrm{P}<0.05$ was considered to indicate a statistically significant difference.

\section{Results}

Proteasome inhibition reduces gallstone formation in mice. To examine the role of proteasome inhibition on gallstone formation, C57BL/6J mice were intraperitoneally injected with BT or CF $(0.5 \mathrm{mg} / \mathrm{kg} /$ week $)$ and fed a lithogenic diet for 12 weeks. After 12 weeks, 8 out of the 10 mice that were fed a lithogenic diet, exhibited gallstone formation, whereas none of the mice that were fed a lithogenic diet and were treated with BT or CF displayed gallstone formation (Fig. 1A). As
Table I. Primers used for quantitative polymerase chain reaction.

Genes Primer sequences

$\begin{array}{ll}\begin{array}{l}\text { srebp-2 } \\ \text { (mouse) }\end{array} & \text { F: 5'-ACTGACCAGCACCCATACTC-3' } \\ \text { Rmgcr } & \text { 5'-CAGGAGGAGAGTTGGAACCA-3' } \\ \text { (mouse) } & \text { F: 5'-ATCTCCTCTCCACAAAGCTT-3' } \\ \text { cyp7b1 } & \text { R: 5'-CATTCTCACAGCAAGCTCCC-3' } \\ \text { (mouse) } & \text { F: 5'-GCATCATCCGAGAAGTGCAG-3' } \\ \text { cyp7al } & \text { F: 5'-ATGAGTGGAGGAAAGAGGGC-3' } \\ \text { (mouse) } & \text { R: 5'-ACAGCGTTAGATATCCGGCT-3' } \\ \text { cyp27al } & \text { F: 5'-GAGAGTGAATCAGGGGACCA-3' } \\ \text { (mouse) } & \text { R: 5'-TCAGGAATGGAGGGTTTCAG-3' } \\ \text { cyp8b1 } & \text { F: 5'-AGATTGCAGCGTCTCTTCCAT-3' } \\ \text { (mouse) } & \text { R: 5'-CCTTGCTCCCTCAGAAACTG-3' } \\ \text { bsep } & \text { F: 5'-GGGTTCTACAGGGGTTGGAA-3' } \\ \text { (abcb11) } & \text { R: 5'-GTGAACTTGGCCACACTCAG-3' }\end{array}$

(mouse)

$m d r 2$

(abcb4)

F: 5'-TCGCAGAGAACATCGCCTAT-3'

(mouse)

$\operatorname{abcg} 5$

R: 5'-TCTCGATGAAGGGGTGGATG-3'

(mouse)

abcg8

(mouse)

fxr

(nr1h4)

(mouse)

pxr

(nrli2)

(mouse)

shp

(nrOb2)

(mouse)

lxr $\alpha$

(nrlh3)

(mouse)'

lxr $\beta$

(nrlh2)

(mouse)

gapdh $^{\mathrm{a}}$

(mouse)
F: 5'-AATTTTGGGGGAATTTCCAG-3'
R: 5'-GTCCTGTGGTTGGCTCATCT-3'
F: 5'-CCTGATCCGTCGTCAGATTT-3'
R: 5'-CCATGGCCGTAGTAAAGGAA-3'
F: 5'-TGGGTACCAGGGAGAGACTG-3'
R: 5'-GTGAGCGCGTTGTAGTGGTA-3'

F: 5'-CCCATCAACGTAGAGGAGGA-3' R: 5'-TCTGAAAAACCCCTTGCATC-3'

F: 5'-AGCTGGGTCCCAAGGAGTAT-3' R: 5'-GGTACCAGGGCTCCAAGACT-3'

\section{F: 5'-TACGTCTCCATCAACCACCC-3'}

R: 5'-CTTGCTCTGAATGGACGCTG-3'

F: 5'-CAGACGCTACAACCACGAGA-3' R: 5'-ATGAATTCCACCTGCAAGCC-3'

\section{F: 5'-CGACTTCAACAGCAACTCCCAC TCTTCC-3' \\ R: 5'-TGGGTGGTCCAGGGTTTCTTA CTCCTT-3'}

a(39). SREBP-2, sterol regulatory element-binding protein-2; HMGCR, 3-hydroxy-3-methylglutaryl-CoA reductase; Cyp7b1, oxysterol $7 \alpha$-hydroxylase; Cyp7a1, cholesterol $7 \alpha$-hydroxylase; Cyp27a1, sterol 27-hydroxylase; Cyp8b1, sterol 12 $\alpha$-hydroxylase; BSEP, bile salt export pump; MDR2, multidrug resistance 2; ABCG, ATP-binding cassette transporter; FXR, farnesoid X receptor; PXR, pregnane X receptor; SHP, small heterodimer partner; LXR, liver X receptor, GAPDH, glyceraldehyde 3-phosphate dehydrogenase; $F$, forward; $R$, reverse.

expected, serum cholesterol and bile acid levels increased after 12 weeks on a lithogenic diet, but proteasome inhibition with BT or CF significantly reduced these levels (Fig. 1B and C). 

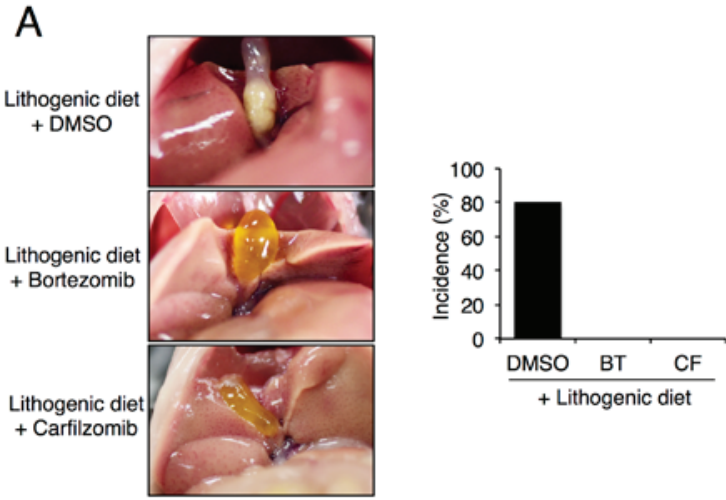

B

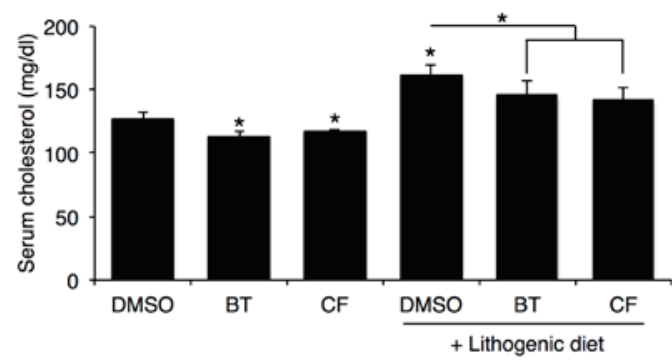

C

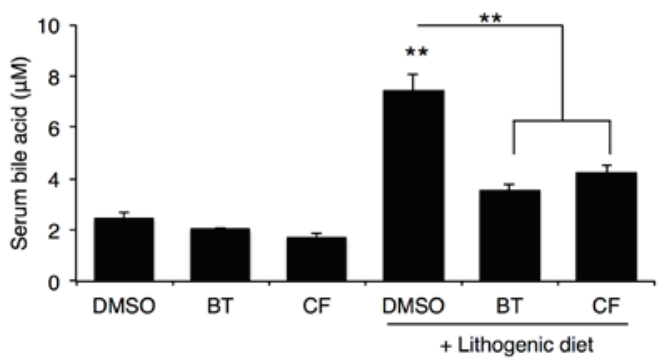

Figure 1. Proteasome inhibition reduces gallstone formation induced by a lithogenic diet. C57BL/6J mice were fed a lithogenic diet for 12 weeks and were administered intraperitoneal injections of BT $(0.5 \mathrm{mg} / \mathrm{kg} /$ week $)$ or CF $(0.5 \mathrm{mg} / \mathrm{kg} /$ week). (A) Image of gallstone formation (left panel) and incidence (right panel) $(\mathrm{n}=10)$. (B) Serum cholesterol and (C) bile acid levels were measured with colorimetric kits $(n=3)$. Data are expressed as the mean \pm standard error of the mean. ${ }^{*} \mathrm{P}<0.05$ and ${ }^{* *} \mathrm{P}<0.01$. Three independent experiments were performed. DMSO, dimethyl sulfoxide; BT, bortezomib; $\mathrm{CF}$, carfilzomib.

Proteasome inhibition reduces cholesterol and bile acid synthesis in the liver. To investigate the protective mechanism of proteasome inhibition on gallstone formation, the hepatic cholesterol levels and expression of genes involved in cholesterol synthesis were analyzed. In accordance with serum cholesterol levels, hepatic cholesterol levels that were elevated with a lithogenic diet were significantly reduced upon BT or CF administration (Fig. 2A). Proteasome inhibition reduced the mRNA and protein levels of Srebp-2, the master regulator of cholesterol synthesis, and $\mathrm{Hmgcr}$, the rate-limiting enzyme of cholesterol synthesis (Fig. 2B and C) (4).

Next, the hepatic bile acid levels and expression of genes involved in bile acid synthesis were examined. Proteasome inhibition reduced the bile acid levels in the liver that were elevated by the lithogenic diet (Fig. 3A). Proteasome inhibition also reduced mRNA and protein expression of hepatic genes involved in bile acid synthesis, including Cyp7al, Cyp7b1, Cyp27al and Cyp8b1 (Fig. 3B and C).
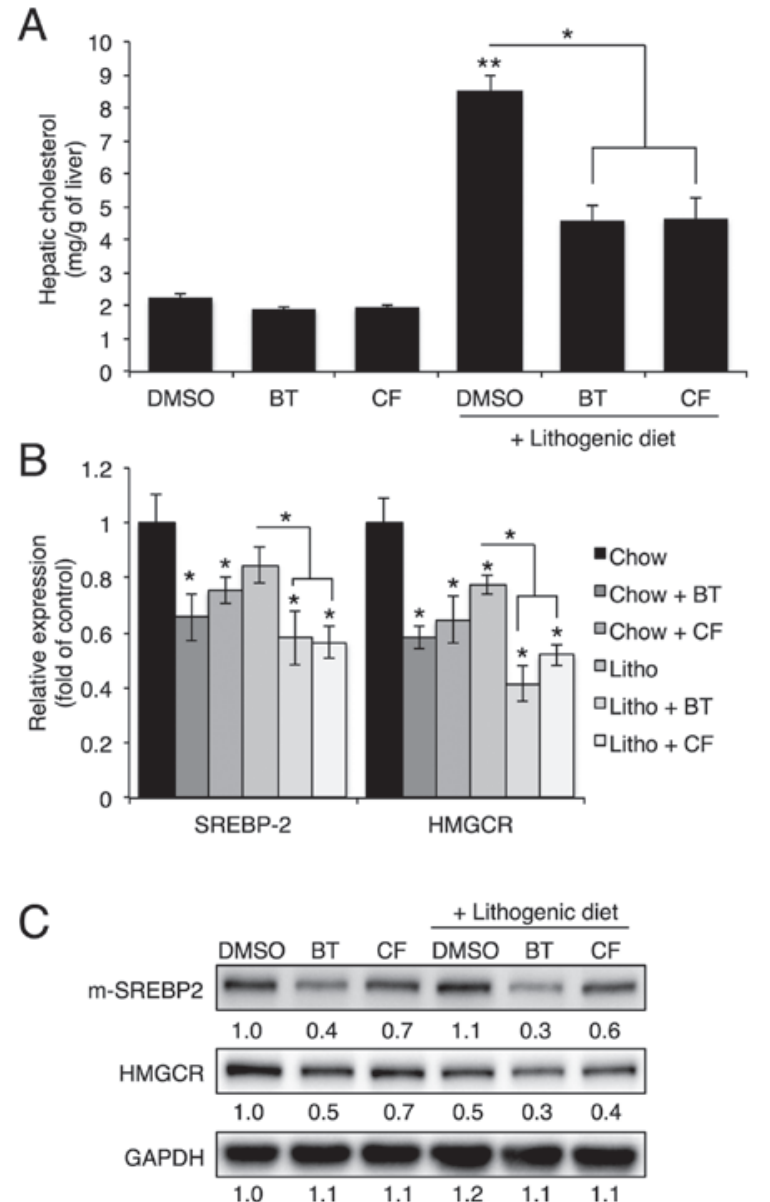

Figure 2. Proteasome inhibition reduces cholesterol synthesis by reducing SREBP-2 and HMGCR expression. (A) Total hepatic cholesterol level in mice injected with BT or CF was quantified with commercial kits (n=3). (B) mRNA levels of SREBP-2 and HMGCR in the livers of mice injected with BT or CF were measured by quantitative polymerase chain reaction $(n=3)$. (C) Representative western blot analyses of SREBP-2 and HMGCR. Densitometric values for each band are provided below the images. Data are expressed as the mean \pm standard error of the mean. ${ }^{*} \mathrm{P}<0.05$ and ${ }^{* *} \mathrm{P}<0.01$. Three independent experiments were performed. BT, bortezomib; CF, carfilzomib; m-SREBP-2, mature form of sterol regulatory element-binding protein-2; HMGCR, 3-hydroxy-3-methylglutaryl-CoA reductase; GAPDH, glyceraldehyde 3-phosphate dehydrogenase; DMSO, dimethyl sulfoxide; Litho, lithogenic diet.

Proteasome inhibition reduces biliary cholesterol secretion. To investigate the effects of proteasome inhibition on biliary secretion, bile composition was further analyzed using commercial kits. Abcg5/Abcg8 heterodimers, Bsep and Mdr2 are crucial for the biliary secretion of cholesterol, bile acids and phospholipids, respectively (6). Notably, BT and CF treatments reduced biliary cholesterol levels, but not biliary phospholipid or bile acid levels (Fig. 4A), suggesting that proteasome inhibition alters only the biliary secretion of cholesterol. In accordance with reduced biliary cholesterol levels, Abcg5 and Abcg 8 mRNA and protein levels were downregulated upon BT or CF injection, whereas Bsep and Mdr2 levels were not significantly affected (Fig. 4B and C). These data indicate that proteasome inhibition of Abcg5 and Abcg8 expression results in reduced biliary secretion of cholesterol and prevents gallstone formation.

Proteasome inhibition induces JNK phosphorylation, which regulates Cyp7al expression. Cholesterol $7 \alpha$-hydroxylase, 

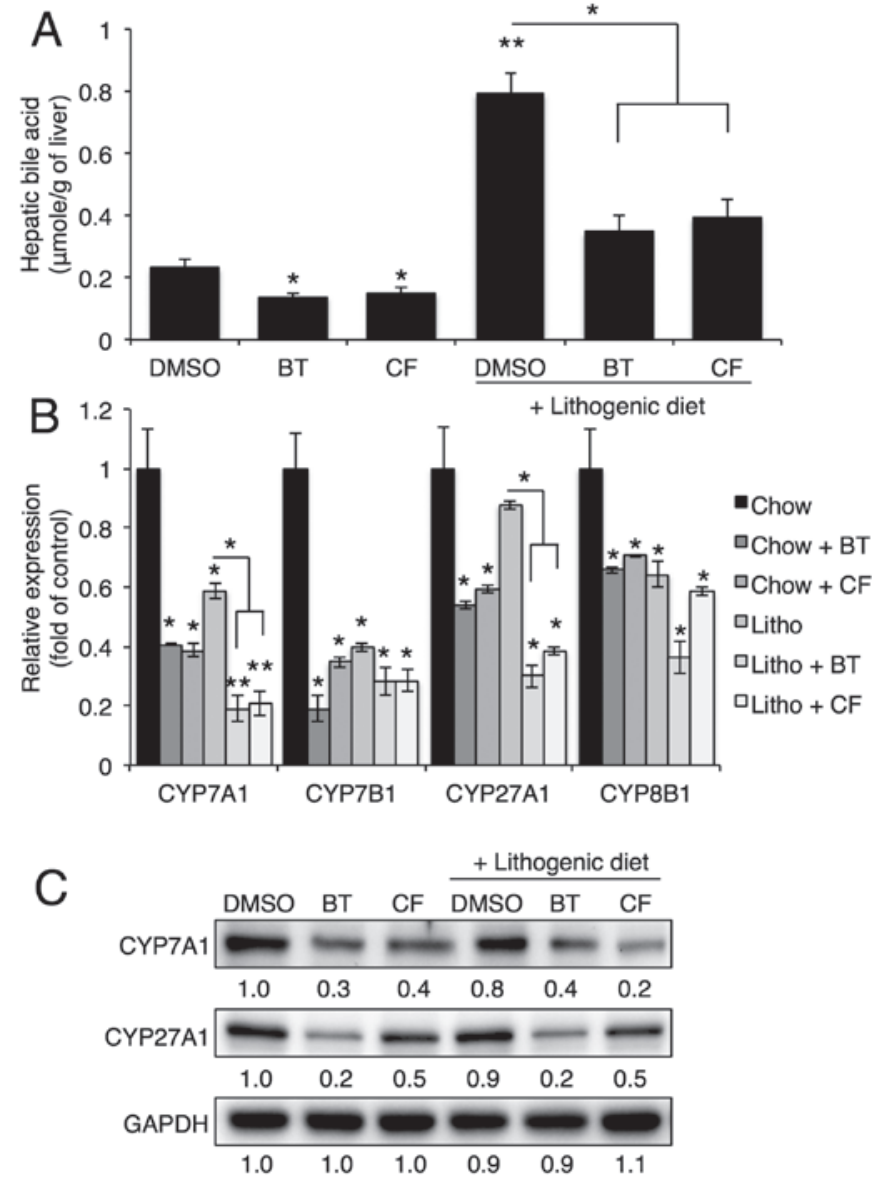

Figure 3. Proteasome inhibition reduces bile acid synthesis by reducing Cyp7a1, Cyp7b1, Cyp27a1 and Cyp8b1 expression. (A) Hepatic bile acid levels upon BT or CF injection were quantified with commercial kits $(\mathrm{n}=3)$. (B) mRNA levels of Cyp7a1, Cyp7b1, Cyp27a1 and Cyp8b1 in the livers of mice injected with BT or CF were measured by quantitative polymerase chain reaction $(n=3)$. (C) Representative western blot analyses of Cyp7a1 and Cyp27a1. Densitometric values for each band are provided below the images. Data are expressed as the mean \pm standard error of the mean. ${ }^{*} \mathrm{P}<0.05$ and ${ }^{* *} \mathrm{P}<0.01$. Three independent experiments were performed. BT, bortezomib; CF, carfilzomib; GAPDH, glyceraldehyde 3-phosphate dehydrogenase; DMSO, dimethyl sulfoxide; CYP7B1, oxysterol 7 $\alpha$-hydroxylase; CYP7A1, cholesterol 7 $\alpha$-hydroxylase; CYP27A1, sterol 27-hydroxylase; CYP8B1, sterol 12 $\alpha$-hydroxylase; Litho, lithogenic diet.

which is encoded by Cyp7al, catalyzes the initial rate-limiting step in cholesterol catabolism (5), and Cyp7al transcription has been shown to be inhibited by the transcription factors SHP, FXR and PXR (5). To examine whether these transcription factors are involved in the reduction of Cyp7a1 levels as a result of proteasome inhibition, SHP, FXR and PXR levels were examined upon treatment with proteasome inhibitors. As they have been shown to inhibit Cyp7al transcription, SHP, FXR and PXR levels were expected to increase upon proteasome inhibition; however, SHP, FXR and PXR levels were reduced in livers treated with proteasome inhibitors (Fig. 5A). Thus it was concluded that SHP, FXR and PXR do not serve roles in the proteasome inhibition-induced reduction of Cyp7a1.

As mitogen-activated protein kinase (MAPK) has also been shown to regulate Cyp7al transcription (22-24), the
A

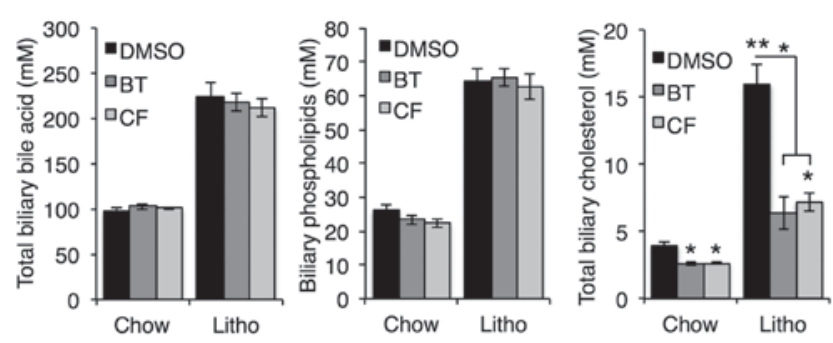

B

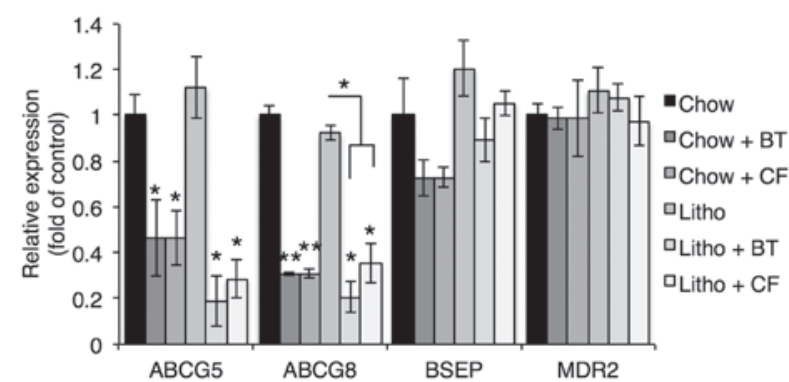

C

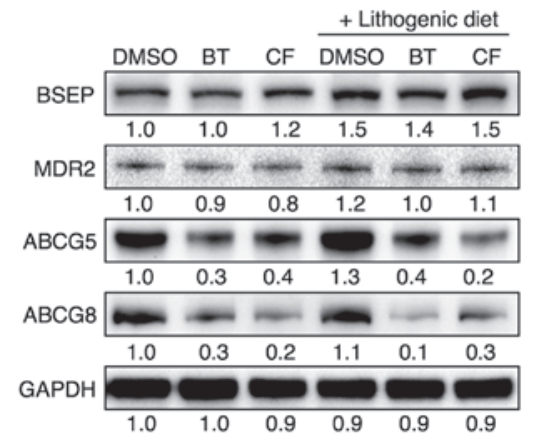

Figure 4. Proteasome inhibition reduces cholesterol secretion by reducing expression of Abcg5 and Abcg8. (A) Biliary cholesterol, bile acid and phospholipid levels were measured in mice injected with BT or CF. (B) mRNA levels of ABCG5, ABCG8, BSEP and MDR2 in the livers of mice injected with $\mathrm{BT}$ or $\mathrm{CF}$ were measured by quantitative polymerase chain reaction $(n=3)$. (C) Representative western blot analyses of BSEP, MDR2, ABCG5 and ABCG8. Densitometric values for each band are provided below the images. Data are expressed as the mean \pm standard error of the mean. ${ }^{~} \mathrm{P}<0.05$, ${ }^{* *} \mathrm{P}<0.01$ and ${ }^{* * *} \mathrm{P}<0.001$. Three independent experiments were performed. BT, bortezomib; CF, carfilzomib; GAPDH, glyceraldehyde 3-phosphate dehydrogenase; DMSO, dimethyl sulfoxide; Litho, lithogenic diet; BSEP, bile salt export pump; MDR2, multidrug resistance protein 2; ABCG, ATP-binding cassette transporter.

phosphorylation of p38, ERK and JNK was examined in livers treated with proteasome inhibitors. Hepatic phosphorylation of JNK and ERK, but not p38, was elevated upon administration of BT and CF (Fig. 5B). To determine whether proteasome inhibition-induced phosphorylation of JNK or of ERK led to the reduced expression of cholesterol and bile acid synthesis genes, the JNK inhibitor SP600125 and the ERK inhibitor PD98059 were used. In accordance with in vivo data, Hep3B cells treated with the proteasome inhibitors BT, CF and Epoxomicin displayed elevated ERK and JNK phosphorylation accompanied by concomitant reductions in mature form of (m)-SREBP2, HMGCR, CYP7A1 and CYP27A1 levels (Fig. 6A). Inhibition of JNK, but not ERK, reversed the downregulation of CYP7A1 and CYP27A1 that was induced by proteasome inhibition, but the proteasome inhibition-induced reduction of 
A

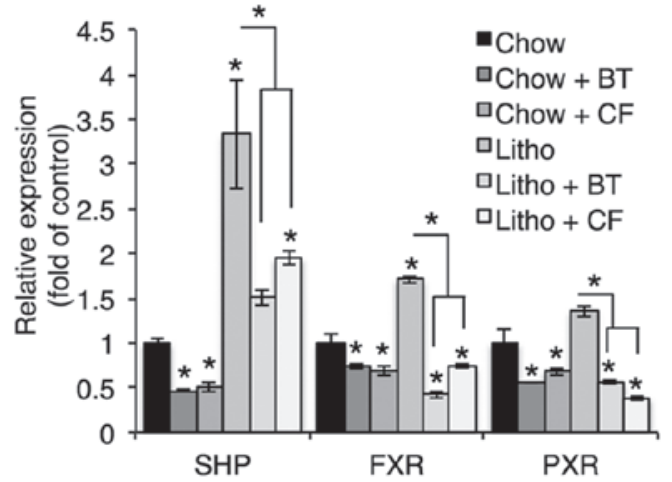

B

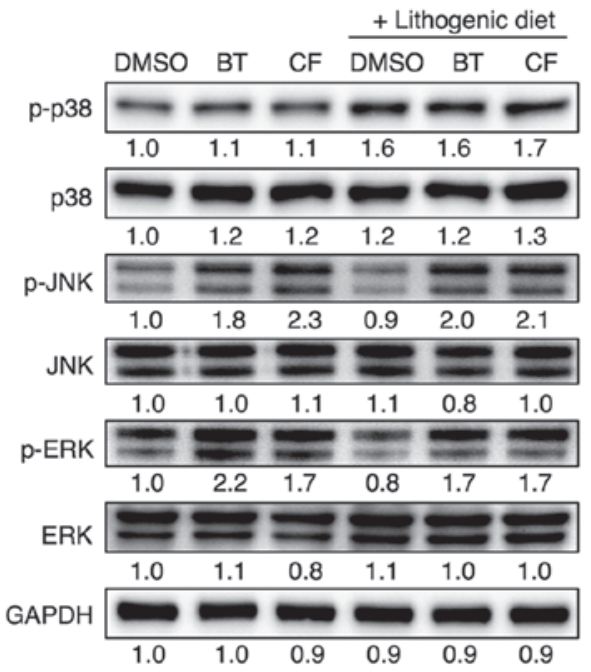

Figure 5. Proteasome inhibition increases phosphorylation of JNK and ERK. (A) mRNA levels of SHP, FXR and PXR in the livers of mice injected with BT or $\mathrm{CF}$ were measured by quantitative polymerase chain reaction $(n=3)$. (B) Representative western blot analyses of proteins in the MAPK pathway. Densitometric values for each band are provided below the images. Data are expressed as the mean \pm standard error of the mean. ${ }^{*} \mathrm{P}<0.05$. Three independent experiments were performed. BT, bortezomib; CF, carfilzomib; GAPDH, glyceraldehyde 3-phosphate dehydrogenase; DMSO, dimethyl sulfoxide; Litho, lithogenic diet; JNK, c-Jun N-terminal protein kinase; ERK, extracellular signal-regulated kinase; MAPK, mitogen-activated protein kinase; SHP, small heterodimer partner; FXR, farnesoid X receptor; PXR, pregnane $\mathrm{X}$ receptor.

A

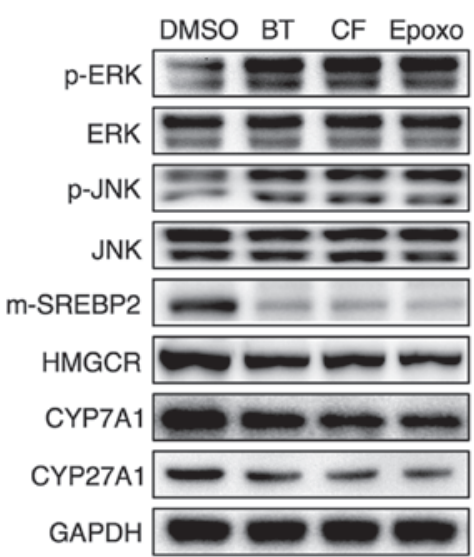

B

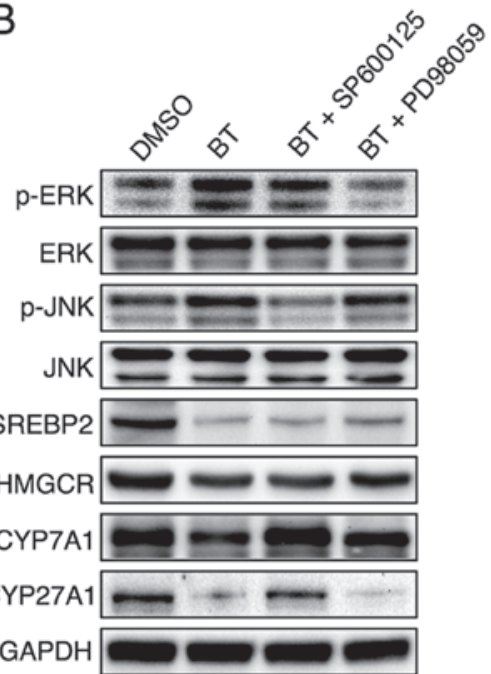

Figure 6. JNK phosphorylation as a result of proteasome inhibition serves a critical role in the downregulation of Cyp7a1 and Cyp27a1. Representative western blot analyses of (A) Hep3B cells treated with $50 \mathrm{nM}$ BT, $50 \mathrm{nM} \mathrm{CF}$ or $50 \mathrm{nM}$ Epoxomicin (Epoxo) and (B) Hep3B cells treated with $50 \mathrm{nM}$ BT and $10 \mu \mathrm{M}$ SP600125 (JNK inhibitor) or $10 \mu \mathrm{M}$ PD98059 (ERK inhibitor). Three independent experiments were performed. BT, bortezomib; CF, carfilzomib; Epoxo, epoxomicin; GAPDH, glyceraldehyde 3-phosphate dehydrogenase; DMSO, dimethyl sulfoxide; JNK, c-Jun N-terminal protein kinase; ERK, extracellular signal-regulated kinase; CYP7A1, cholesterol 7 $\alpha$-hydroxylase; CYP27A1, sterol 27-hydroxylase; m-SREBP-2, mature form of sterol regulatory element-binding protein-2; HMGCR, 3-hydroxy-3-methylglutaryl-CoA reductase.

SREBP-2 and HMGCR was not reversed with JNK or ERK inhibitors (Fig. 6B).

Proteasome inhibition reduces ABCG5 and ABCG8 levels by reducing $P K A$ phosphorylation. To evaluate how proteasome inhibitors regulate ABCG5 and ABCG8, the levels of liver $\mathrm{X}$ receptor (LXR) $\alpha$ and $\mathrm{LXR} \beta$, which are known to regulate ABCG5 and ABCG8, were examined in mouse livers in the presence of proteasome inhibitors (25). It was found that LXR $\alpha$ and LXR $\beta$ levels were not affected by proteasome inhibitors, suggesting that the proteasome inhibitor-induced downregulation of ABCG5 and ABCG8 was not caused by LXR $\alpha$ and LXR $\beta$ (Fig. 7A). Thus, the PKA signaling pathway, which has also been shown to affect ABCG5 and ABCG8 expression, was analyzed (26). Reduced PKA phosphorylation was found in Hep3B cells that were treated with proteasome inhibitors (Fig. 7B). It was also found that treatment with a PKA activator, 8-Br-cAMP or Sp-cAMPS, reversed the proteasome inhibition-induced reduction of ABCG5 and ABCG8 levels (Fig. 7C), confirming that PKA phosphorylation serves 
A

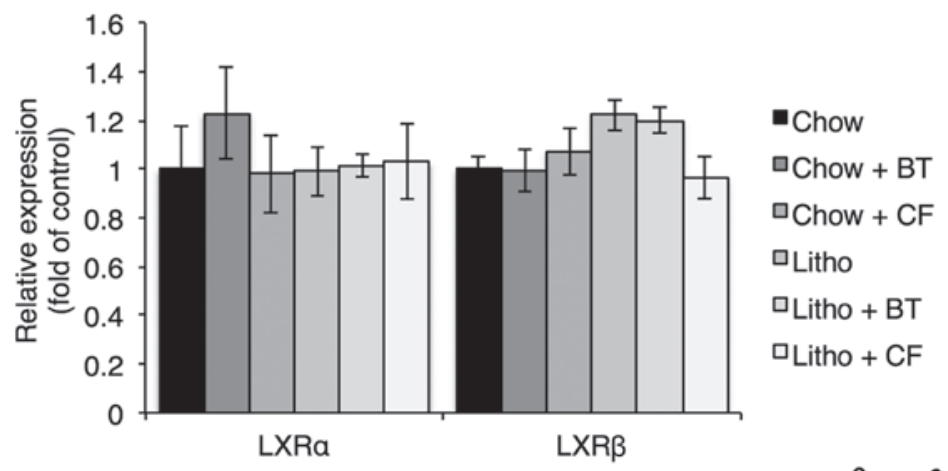

B
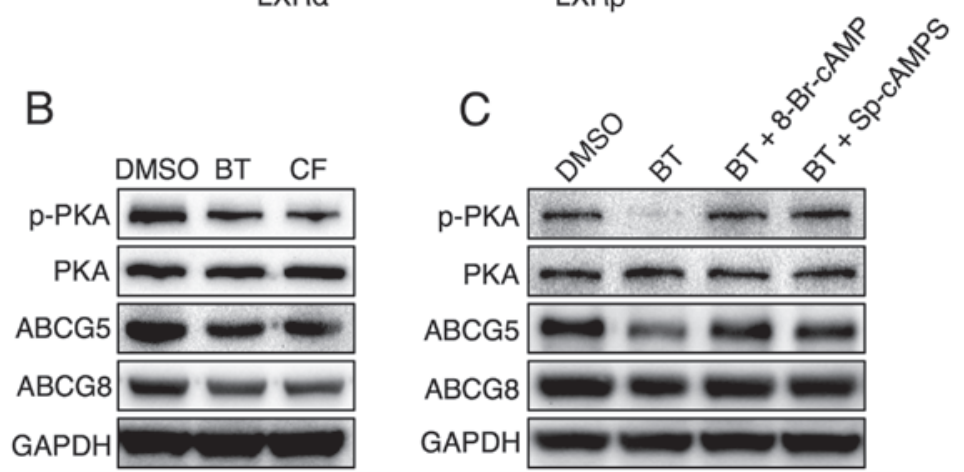

Figure 7. Reduced PKA phosphorylation as a result of proteasome inhibition serves a critical role in the downregulation of ABCG5 and ABCG8. (A) mRNA levels of $\mathrm{LXR} \alpha$ and LXR $\beta$ in the livers of mice injected with BT or CF were measured by quantitative polymerase chain reaction ( $\mathrm{n}=3$ ). Representative western blot analyses of (B) Hep3B cells treated with $50 \mathrm{nM}$ BT or $50 \mathrm{nM} \mathrm{CF}$, and (C) Hep3B cells treated with $50 \mathrm{nM}$ BT and $10 \mu \mathrm{M} 8$-Br-cAMP or Sp-cAMPS (PKA activators). Three independent experiments were performed. BT, bortezomib; CF, carfilzomib; GAPDH, glyceraldehyde 3-phosphate dehydrogenase; DMSO, dimethyl sulfoxide; Litho, lithogenic diet; ABCG, ATP-binding cassette transporter; PKA, protein kinase A; LXR, liver X receptor.

a critical role in the regulation of ABCG5 and ABCG8 levels during proteasome inhibition.

\section{Discussion}

Gallstone disease is one of the most common gastrointestinal diseases worldwide, and the cholesterol, phospholipid and bile acid composition of bile serves an important role in the pathogenesis of cholesterol gallstone formation (2). For example, cholesterol overcomes its low solubility in aqueous solutions by forming micelles with phospholipids and bile acids $(2,3)$. Clinically, the main strategies to prevent gallstone disease have been focused on reducing cholesterol synthesis, reducing cholesterol secretion or inducing FXR, which increases bile acid and phospholipid secretion by increasing BSEP and MDR2 levels (27). In the present study, proteasome inhibition with BT or CF prevented gallstone formation by reducing cholesterol synthesis (via reductions in SREBP-2 and HMGCR) and by reducing cholesterol secretion (via reductions in ABCG5 and ABCG8). As excess cholesterol in bile can precipitate and lead to gallstone formation, reducing biliary cholesterol by proteasome inhibition is a strategy that can be utilized clinically to prevent gallstone disease. Considering that BT is currently utilized to treat hepatic conditions and certain cancer types, the protective effect of BT on gallstone formation has clinical importance.

Hepatic cholesterol levels, hepatic bile acid levels and the expression of genes involved in bile acid synthesis were significantly reduced upon proteasome inhibition. Reduced degradation of cholesterol to bile acids may lead to a higher concentration of free cholesterol that can be secreted leading to cholesterol saturation and gallstone formation (28). However, in contrast to hepatic bile acid levels, biliary bile acid levels were not affected by proteasome inhibition, thus biliary bile acid levels do not appear to affect gallstone formation. Rather, hepatic bile acid synthesis may be reduced to compensate for the reduction in hepatic cholesterol induced by proteasome inhibition.

In the present study, Cyp7a1 and Cyp27a1 protein levels were regulated by JNK phosphorylation as a result of proteasome inhibition. JNK phosphorylation has been shown to be induced by inflammatory cytokines, including tumor necrosis factor- $\alpha$ (TNF- $\alpha)$ and interleukin-1 $\beta$ (IL-1 $\beta)(29,30)$. However, TNF- $\alpha$ and IL-1 $\beta$ levels in the liver and serum were not altered upon proteasome inhibition (data not shown), suggesting that they are not involved in proteasome inhibition-induced phosphorylation of JNK. Although proteasome inhibition has been shown to activate MAPK $(31,32)$, to the best of our knowledge, this is the first study to demonstrate that JNK regulates genes involved in bile acid synthesis upon proteasome inhibition. In contrast to the regulation of Cyp7a1 and Cyp27a1, JNK or ERK inhibition was unable to reverse the reduction in SREBP-2 and HMGCR levels induced by proteasome inhibition. The mechanism of the reduction in SREBP-2 and HMGCR levels by proteasome inhibition remains to be elucidated.

Proteasome inhibition also reduced the hepatic levels of the cholesterol transporters ABCG5 and ABCG8, but did not reduce the hepatic levels of the phospholipid and bile acid transporters MDR2 and BSEP, respectively. These data indicate that proteasome inhibition reduced hepatic cholesterol secretion to 
bile and reduced hepatic cholesterol synthesis. Although LXR activation has been shown to increase gallstone formation by regulating ABCG5 and ABCG8 levels $(25,33)$, BT or CF injection did not affect $\mathrm{LXR} \alpha$ and $\mathrm{LXR} \beta$ levels. PKA has also been shown to regulate ABCG5 and ABCG8 expression (26), and the present study showed that PKA phosphorylation was reduced by proteasome inhibition. In addition, it was found that PKA activation by 8-bromo-cAMP or Sp-cAMPS reversed the reduction of ABCG5 and ABCG8 upon proteasome inhibition, confirming the role of PKA in the proteasome inhibition-induced regulation of ABCG5 and ABCG8.

Although proteolytic activity of the $26 \mathrm{~S}$ proteasome is ATP- and ubiquitin-dependent, c-AMP-induced PKA activation also stimulates the proteolytic activity of the proteasome (34). The regulatory subunit of PKA has been shown to be degraded via the proteasome (35), thus proteasome inhibition would lead to accumulation of the regulatory subunit of PKA. As the regulatory subunit of PKA functions as an inhibitor, PKA phosphorylation would be reduced (36).

BT is currently used clinically as a first-line chemotherapeutic agent for multiple myeloma, and high doses of BT induce endoplasmic reticulum stress and cell death $(12,15,37)$. In addition, high doses of BT often lead to side effects, such as weight loss and painful neuropathy (38). It has been shown that administration of a low dose of BT $(0.5 \mathrm{mg} / \mathrm{kg} /$ week $)$ does not increase ER stress (13). Consistent with this, no signs of ER stress were observed with this low BT dose in the present study (data not shown). Although several studies have shown the protective effects of proteasome inhibition for liver diseases, including drug-induced hepatotoxicity (15) and ethanol-induced steatosis (13), to the best of our knowledge, this study is the first to show that proteasome inhibitors could be used to prevent gallstone formation.

In conclusion, in the present study, proteasome inhibitors reduced hepatic cholesterol and bile acid synthesis, and reduced biliary cholesterol secretion by reducing expression of the cholesterol transporters ABCG5 and ABCG8. This finding may lead to novel strategies for the treatment of gallstone disease.

\section{Acknowledgements}

This study was supported by the Gachon University Research Fund of 2013 (grant no. 2013-M050).

\section{References}

1. Stinton LM and Shaffer EA: Epidemiology of gallbladder disease: cholelithiasis and cancer. Gut Liver 6: 172-187, 2012.

2. Kosters A, Jirsa M and Groen AK: Genetic background of cholesterol gallstone disease. Biochim Biophys Acta 1637: $1-19,2003$.

3. Portincasa P, Moschetta A and Palasciano G: Cholesterol gallstone disease. Lancet 368: 230-239, 2006.

4. Tao R, Xiong X, DePinho RA, Deng CX and Dong XC: Hepatic SREBP-2 and cholesterol biosynthesis are regulated by FoxO3 and Sirt6. J Lipid Res 54: 2745-2753, 2013.

5. Chiang JY: Bile acids: regulation of synthesis. J Lipid Res 50: 1955-1966, 2009.

6. Van Erpecum KJ: Pathogenesis of cholesterol and pigment gallstones: an update. Clin Res Hepatol Gastroenterol 35: 281-287, 2011.

7. Ahmed MH, Hamad MA, Routh C and Connolly V: Statins as potential treatment for cholesterol gallstones: an attempt to understand the underlying mechanism of actions. Expert Opin Pharmacother 12: 2673-2681, 2011.
8. Merzon E, Weiss NS, Lustman AJ, Elhayani A, Dresner J and Vinker S: Statin administration and risk of cholecystectomy: a population-based case-control study. Expert Opin Drug Saf 9: 539-543, 2010.

9. Cariati A and Piromalli E: Limits and perspective of oral therapy with statins and aspirin for the prevention of symptomatic cholesterol gallstone disease. Expert Opin Pharmacother 13: 1223-1227, 2012.

10. Kotb MA: Molecular mechanisms of ursodeoxycholic acid toxicity \& side effects: ursodeoxycholic acid freezes regeneration \& induces hibernation mode. Int J Mol Sci 13: 8882-8914, 2012.

11. Adams J, Palombella VJ and Elliott PJ: Proteasome inhibition: a new strategy in cancer treatment. Invest New Drugs 18: 109-121, 2000.

12. Field-Smith A, Morgan GJ and Davies FE: Bortezomib (Velcadetrade mark) in the treatment of multiple myeloma. Ther Clin Risk Manag 2: 271-279, 2006.

13. Oliva J, French SW, Li J and Bardag-Gorce F: Proteasome inhibitor treatment reduced fatty acid, triacylglycerol and cholesterol synthesis. Exp Mol Pathol 93: 26-34, 2012.

14. Anan A, Baskin-Bey ES, Isomoto H, Mott JL, Bronk SF, Albrecht $\mathrm{JH}$ and Gores GJ: Proteasome inhibition attenuates hepatic injury in the bile duct-ligated mouse. Am J Physiol Gastrointest Liver Physiol 291: G709-G716, 2006.

15. Park WJ, Kim SY, Kim YR and Park JW: Bortezomib alleviates drug-induced liver injury by regulating CYP2E1 gene transcription. Int J Mol Med 37: 613-622, 2016.

16. Nawrocki ST, Carew JS, Pino MS, Highshaw RA, Dunner K Jr, Huang P, Abbruzzese JL and McConkey DJ: Bortezomib sensitizes pancreatic cancer cells to endoplasmic reticulum stress-mediated apoptosis. Cancer Res 65: 11658-11666, 2005.

17. Codony-Servat J, Tapia MA, Bosch M, Oliva C, Domingo-Domenech J, Mellado B, Rolfe M, Ross JS, Gascon P, Rovira A, et al: Differential cellular and molecular effects of bortezomib, a proteasome inhibitor, in human breast cancer cells. Mol Cancer Ther 5: 665-675, 2006.

18. Kondagunta GV, Drucker B, Schwartz L, Bacik J, Marion S, Russo P, Mazumdar M and Motzer RJ: Phase II trial of bortezomib for patients with advanced renal cell carcinoma. J Clin Oncol 22: 3720-3725, 2004.

19. Papandreou CN, Daliani DD, Nix D, Yang H, Madden T, Wang X, Pien CS, Millikan RE, Tu SM, Pagliaro L, et al: Phase I trial of the proteasome inhibitor bortezomib in patients with advanced solid tumors with observations in androgen-independent prostate cancer. J Clin Oncol 22: 2108-2121, 2004.

20. Dick LR and Fleming PE: Building on bortezomib: second-generation proteasome inhibitors as anti-cancer therapy. Drug Discov Today 15: 243-249, 2010.

21. Livak KJ and Schmittgen TD: Analysis of relative gene expression data using real-time quantitative PCR and the 2(-Delta Delta C(T)) method. Methods 25: 402-408, 2001.

22. Wooton-Kee CR, Coy DJ, Athippozhy AT, Zhao T, Jones BR and Vore M: Mechanisms for increased expression of cholesterol 7alpha-hydroxylase (Cyp7a1) in lactating rats. Hepatology 51: 277-285, 2010.

23. Gupta S, Stravitz RT, Dent P and Hylemon PB: Down-regulation of cholesterol 7alpha-hydroxylase (CYP7A1) gene expression by bile acids in primary rat hepatocytes is mediated by the c-Jun N-terminal kinase pathway. J Biol Chem 276: 15816-15822, 2001.

24. Stroup D: Kinase/phosphatase regulation of CYP7A1. Front Biosci 10: 1678-1692, 2005.

25. Repa JJ, Berge KE, Pomajzl C, Richardson JA, Hobbs H and Mangelsdorf DJ: Regulation of ATP-binding cassette sterol transporters ABCG5 and ABCG8 by the liver X receptors alpha and beta. J Biol Chem 277: 18793-18800, 2002.

26. Yamazaki Y, Yasui K, Hashizume T, Suto A, Mori A, Murata Y, Yamaguchi M, Ikari A and Sugatani J: Involvement of a cyclic adenosine monophosphate-dependent signal in the diet-induced canalicular trafficking of adenosine triphosphate-binding cassette transporter g5/g8. Hepatology 62: 1215-1226, 2015.

27. Moschetta A, Bookout AL and Mangelsdorf DJ: Prevention of cholesterol gallstone disease by FXR agonists in a mouse model. Nat Med 10: 1352-1358, 2004.

28. Bertolotti M, Gabbi C, Anzivino C, Carulli L and Carulli N: Changes in bile acid synthesis in gallstone disease: cause, consequence, or neither? Hepatology 46: 1664-1665, 2007.

29. Li T, Jahan A and Chiang JY: Bile acids and cytokines inhibit the human cholesterol 7 alpha-hydroxylase gene via the JNK/c-jun pathway in human liver cells. Hepatology 43: 1202-1210, 2006. 
30. Liu X, Qi Y, Tian B, Chen D, Gao H, Xi C, Xing Y and Yuan Z Maternal protein restriction induces alterations in hepatic tumor necrosis factor- $\alpha /$ CYP7A1 signaling and disorders regulation of cholesterol metabolism in the adult rat offspring. J Clin Biochem Nutr 55: 40-47, 2014.

31. Choi $\mathrm{CH}$, Lee $\mathrm{BH}$, Ahn $\mathrm{SG}$ and $\mathrm{Oh} \mathrm{SH}$ : Proteasome inhibition-induced 38 MAPK/ERK signaling regulates autophagy and apoptosis through the dual phosphorylation of glycogen synthase kinase 33. Biochem Biophys Res Commun 418: 759-764, 2012.

32. Wang HQ, Liu BQ, Gao YY, Meng X, Guan Y, Zhang HY and Du ZX: Inhibition of the JNK signalling pathway enhances proteasome inhibitor-induced apoptosis of kidney cancer cells by suppression of BAG3 expression. Br J Pharmacol 158: 1405-1412, 2009.

33. Uppal H,Zhai Y, Gangopadhyay A, Khadem S, Ren S, Moser JA and Xie W: Activation of liver X receptor sensitizes mice to gallbladder cholesterol crystallization. Hepatology 47: 1331-1342, 2008.

34. Zhang F, Hu Y, Huang P, Toleman CA, Paterson AJ and Kudlow JE: Proteasome function is regulated by cyclic AMP-dependent protein kinase through phosphorylation of Rpt6. J Biol Chem 282: 22460-22471, 2007.
35. Hegde AN, Goldberg AL and Schwartz JH: Regulatory subunits of cAMP-dependent protein kinases are degraded after conjugation to ubiquitin: a molecular mechanism underlying long-term synaptic plasticity. Proc Natl Acad Sci USA 90: 7436-7440, 1993.

36. Lin JT, Chang WC, Chen HM, Lai HL, Chen CY, Tao MH and Chern Y: Regulation of feedback between protein kinase A and the proteasome system worsens Huntington's disease. Mol Cell Biol 33: 1073-1084, 2013.

37. Takenokuchi M, Miyamoto $\mathrm{K}$, Saigo $\mathrm{K}$ and Taniguchi $\mathrm{T}$ : Bortezomib causes ER stress-related death of acute promyelocytic leukemia cells through excessive accumulation of PML-RARA. Anticancer Res 35: 3307-3316, 2015.

38. Meregalli C, Carozzi VA, Sala B, Chiorazzi A, Canta A, Oggioni N, Rodriguez-Menendez V, Ballarini E, Ceresa C, Nicolini G, et al: Bortezomib-induced peripheral neurotoxicity in human multiple myeloma-bearing mice. J Biol Regul Homeost Agents 29: 115-124, 2015 .

39. Liu G, Friggeri A, Yang Y, Park Y-J, Tsuruta Y and Abraham E: miR-147, a microRNA that is induced upon Toll-like receptor stimulation, regulates murine macrophage inflammatory responses. Proc Natl Acad Sci USA 106: 15819-15824, 2009. 